\title{
Dynamic behaviors of a broad-area diode laser with lateral-mode-selected external feedback
}

\section{Chi, Mingjun; Petersen, Paul Michael}

\section{Published in:}

Proceedings of the SPIE

Link to article, DOI:

$10.1117 / 12.2071842$

Publication date:

2014

Document Version

Publisher's PDF, also known as Version of record

Link back to DTU Orbit

Citation (APA):

Chi, M., \& Petersen, P. M. (2014). Dynamic behaviors of a broad-area diode laser with lateral-mode-selected external feedback. In Proceedings of the SPIE (Vol. 9267). [92670B] SPIE - International Society for Optical Engineering. Proceedings of SPIE - The International Society for Optical Engineering https://doi.org/10.1117/12.2071842

\section{General rights}

Copyright and moral rights for the publications made accessible in the public portal are retained by the authors and/or other copyright owners and it is a condition of accessing publications that users recognise and abide by the legal requirements associated with these rights.

- Users may download and print one copy of any publication from the public portal for the purpose of private study or research.

- You may not further distribute the material or use it for any profit-making activity or commercial gain

- You may freely distribute the URL identifying the publication in the public portal 


\title{
Dynamic behaviors of a broad-area diode laser with lateral-mode- selected external feedback

\author{
DTU Fotonik, Department of Photonics Engineering, Technical University of Denmark, \\ Frederiksborgvej 399, P.O. Box 49, DK-4000 Roskilde, Denmark
} \\ Mingjun Chi, and Paul Michael Petersen
}

\begin{abstract}
In this paper, we investigate the dynamics of a BAL with lateral-mode selected external feedback experimentally by measuring the far-field profile, intensity noise spectrum and time series of the output beam. The mode-selection is achieved by adjusting a stripe mirror at the pseudo far-field plane. Different dynamic behaviors are observed when different lateral modes are selected. When the mirror is aligned correctly and high-order modes are selected, in most of the cases periodic dynamics of the output power corresponding to a single roundtrip external-cavity loop is observed, but the dynamic behavior disappears in some case; when the zero-order mode is selected, periodic dynamics corresponding to a double roundtrip external-cavity loop is observed. When the stripe mirror is not aligned perfectly, a dynamic behavior like pulse-package oscillations is observed: a periodic oscillated output with a frequency of the single roundtrip external-cavity loop modulated by periodic low-frequency fluctuation. This is the first observation of pulse-package oscillation in a diode laser with long-cavity feedback, to our knowledge.
\end{abstract}

Keywords: Broad-area diode laser, external feedback, laser dynamics, pulse-package oscillation

\section{INTRODUCTION}

Broad-area diode lasers (BALs) are promising light sources for various applications due to their high output power, high electro-optical efficiency, long lifetimes, compactness and low cost. However, BALs suffer from poor spatial beam quality in the slow axis owing to the broad emitter aperture in the slow axis (lateral direction). Different techniques have been developed to improve the beam quality of BALs in the slow axis. Among them, external-cavity feedback with lateral-mode selection is an effective technique to improve the spatial coherence of BALs in lateral direction ${ }^{1-5}$. This external-cavity feedback technique is based on the fact that the output of a gain-guided BAL consists of a set of lateral spatial modes; each high-order mode has a double-lobed far-field in the slow axis, and these modes are distinguished at different angles in the far field ${ }^{6,7}$. By this technique, one lobe of a lateral-mode of the BAL at the far-field is selected and reflected back into the BAL, and the other lobe of the same lateral-mode is coupled out of the laser cavity as output beam of the diode laser system. All the other lateral modes are suppressed effectively, thus the spatial beam quality of a BAL in the slow axis is improved significantly ${ }^{1-5,7}$.

When external feedback is applied to a diode laser, complex dynamics of the output beam will take place. The complex dynamic behaviors have been studied intensively for both narrow-stripe diode lasers ${ }^{8-11}$ and BALs ${ }^{12-19}$ with either a conventional mirror or a phase conjugator external feedback. The dynamic behaviors of BALs have been studied with short-cavity feedback ${ }^{15,16,19}$, long-cavity feedback ${ }^{14,17}$, and tilt mirror feedback ${ }^{12,18}$. Different dynamic behaviors, such as low frequency fluctuations (LFFs) ${ }^{14,17}$, pulse-package oscillations ${ }^{16,19}$, have been observed in BALs subjected to external feedback. Wolff et al investigated the time-resolved lateral near- and far-field distribution of BAL with lateral-modeselected external-cavity feedback numerically, and a self-Q-switched-like mode was observed in a BAL, which was operated high above the threshold ${ }^{1}$. Pimenov et al studied the dynamical regimes of multistripe laser array with external off-axis feedback theoretically, different periodic and aperiodic oscillations were shown in the study ${ }^{20}$. Recently, we investigated the spectral properties of a BAL with off-axis external-cavity feedback ${ }^{21}$, and we found that a four-wave mixing process in the gain medium is responsible for the establishment of the external cavity between the feedback mirror and the BAL. However, the dynamic behavior of BAL subjected to lateral-mode-selected external-cavity feedback has not been investigated systematically.

In this paper, the dynamic behaviors of a BAL with lateral-mode selected external feedback are investigated experimentally. Different dynamics are observed when different lateral modes are selected. When the mirror is aligned correctly and high-order modes are selected, in most of the cases periodic dynamics corresponding to a single roundtrip

Semiconductor Lasers and Applications VI, edited by Ninghua Zhu,

Werner H. Hofmann, Proc. of SPIE Vol. 9267, 92670B - (c) 2014 SPIE

CCC code: $0277-786 \mathrm{X} / 14 / \$ 18 \cdot$ doi: $10.1117 / 12.2071842$

Proc. of SPIE Vol. 9267 92670B-1 
external-cavity loop is observed, but the dynamic behavior disappears in some case; when the zero-order mode is selected, dynamics corresponding to a double roundtrip external-cavity loop is observed. When the stripe mirror is not aligned perfectly, pulse-package oscillation is observed.

\section{EXPERIMENTAL DETAILS}

The experimental setup is shown in Fig. 1. The BAL used in the experiment is an $810 \mathrm{~nm}$, broad-area device with an emitter aperture of $1 \mu \mathrm{m} \times 200 \mu \mathrm{m}$ (fast $\times$ slow axis). The laser diode is antireflection coated on the front facet with a reflectivity of around $6 \%$, and high reflection coated on the back facet. The threshold of the BAL is $0.56 \mathrm{~A}$. The output beam is collimated in the fast axis by an aspherical lens of $4.5 \mathrm{~mm}$ focal length. Together with a cylindrical lens of 80 $\mathrm{mm}$ focal length, these two lenses collimate the beam in the slow axis and transform the near field to the far field at the pseudo-far-field plane simultaneously with respect to the slow axis. All lenses are antireflection coated for near-infrared wavelength. At the pseudo-far-field plane, a mirror with a reflectivity of $99 \%$ and a sharp edge combined with a razor blade is used as a mirror stripe. The mirror stripe, which can be translated in the slow-axis direction and tilted around Y axis, is used for lateral-mode selection. The external cavity is formed between the BAL and the mirror stripe ${ }^{21}$. In the experiment, the injected current to the BAL is $0.6 \mathrm{~A}$ (around 1.09 times threshold). The physical length of the external cavity is around $53 \mathrm{~cm}$, in a long-cavity feedback regime.

The far field at the pseudo-far-field plane consists of high-order lateral modes with a double-lobe profile, and 0-order lateral mode with a single-lobe profile at the beam center. For high-order lateral mode selection, one lobe of a mode is selected and reflected back into the gain medium of the BAL, the other lobe (output lobe) of the same spatial mode is the output beam of the laser system. For 0-order lateral mode selection, the single central lobe is selected and reflected back into the BAL, and there is no output beam in this case.

A beam splitter is inserted into the external cavity, and the reflected beam is used as a diagnostic beam. On this beam, the far-field profile of the output beam is recorded by a beam scanner (Photo Inc., model 0180); the intensity signal of the output beam is monitored by a silicon PIN photodiode (Melles Griot, 13DAH001 with a 3-GHz bandwidth), after a high-frequency amplifier (frequency below $1 \mathrm{MHz}$ is blocked), the electronic signal is sent to an electronic spectrum analyzer (band width $1.8 \mathrm{GHz}$, Tektronix 2753P) for intensity noise spectrum measurement, and to a 1-GHz digital oscilloscope (LeCroy, WaveSurfer 104Xs) for monitoring the time series of the signal.

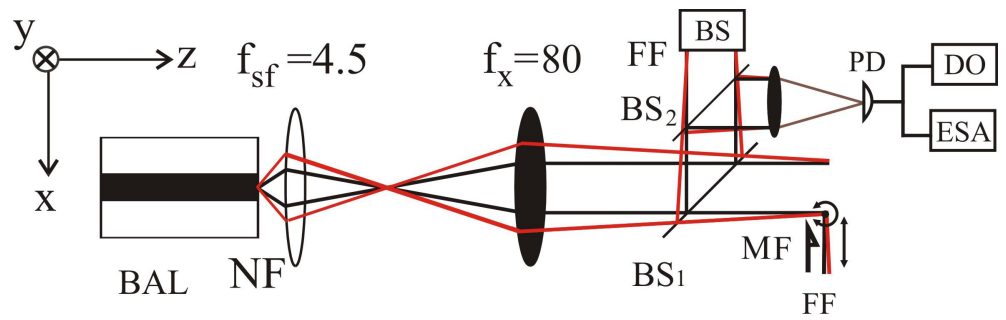

Figure 1. Experimental set-up of the BAL with lateral-mode-selected external-cavity feedback; NF: near field; $\mathrm{BS}_{1}$ and $\mathrm{BS}_{2}$ : beam splitters; FF: far field; MF: mirror with spatial filter. BS: beam scanner; PD: photodiode; DO: digital oscilloscope; ESA: electronic spectrum analyzer. Red lines are for the non-perfect alignment of the mirror.

Here we present the experimental results on two different alignment conditions. The first condition is: The mirror stripe is perfect aligned, this means when a certain lateral mode is selected, the highest output lobe is achieved by adjusting the position and the tilting of the mirror stripe. The second condition is: When the perfect alignment condition is achieved, the mirror stripe is tilted a little bit (in the direction shown as the red lines in Fig.1), the position of the mirror stripe is kept unchanged at the same time, so the feedback beam is injected into the laser cavity with a little larger angle compared with the perfect alignment condition. These two conditions are named as perfect and non-perfect alignment conditions below.

\section{EXPERIMENTAL RESULTS}

First, the experiment is done on the perfect alignment condition. The far-field profiles along the slow axis are measured with and without the external-cavity feedback. The results are shown in Fig. 2, and we shifted the profiles with different mode selections vertically for clarity. Curve a) shows the far-field profile of the freely running laser, and the profile is almost symmetric; curve b) shows the far-field profile when 0 -order lateral-mode is selected, and the profile is also 
almost symmetric; curves c) to g) show the far-field profiles from low-order lateral-mode selection to high-order lateralmode selection, the far-field profiles are highly asymmetric and this is normal for BAL with off-axis external feedback ${ }^{1-}$ 5,7 . The results show that high-order lateral-modes, which do not exist without external feedback, are excited by the lateral-mode selected external-cavity feedback, i.e., curves e) to g). The optical feedback strength, defined as the ratio of the power reflected by the stripe mirror to power towards the feedback mirror, is related to the intensity ratio of the feedback lobe to the total beam, since the reflectivity and the width of the mirror stripe are fixed. When the different lateral-modes are selected, Fig. 2 shows the relative intensity of the feedback lobe to the total beam is different; and this is determined by the laser system itself. The feedback strength decreases from $21.6 \%$ to $7.0 \%$ from curve b) to c), and increases from $4.8 \%$ to $11.6 \%$ from curve d) to g); it is moderate feedback strength for BAL ${ }^{12}$.

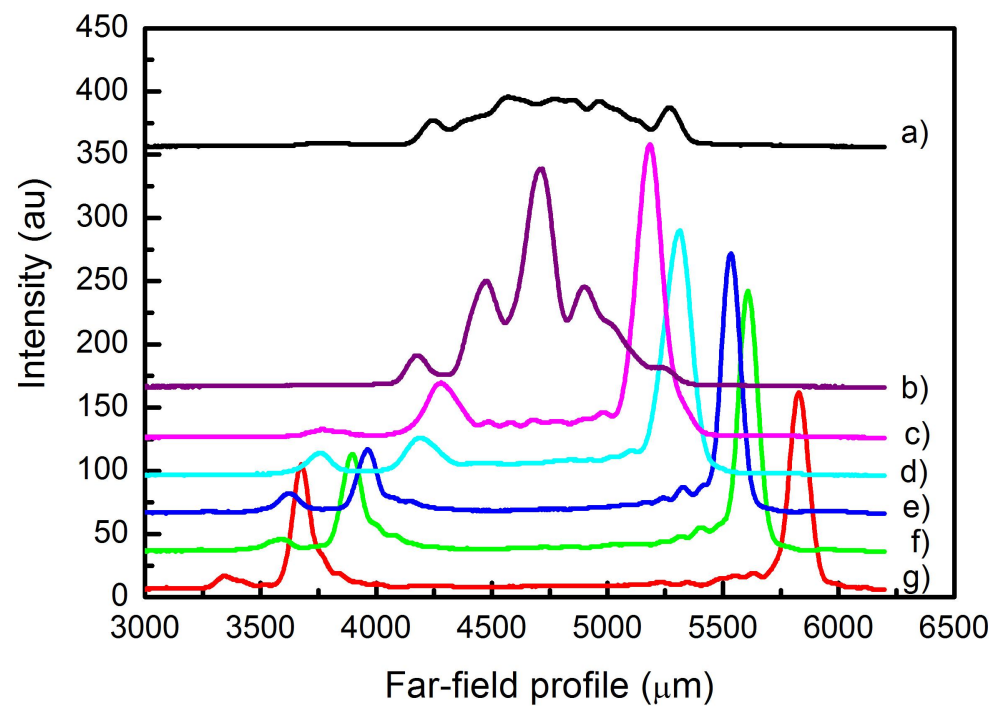

Figure 2. Far-field profiles of the BAL along the slow axis without (black curve) and with (color curves) external-cavity feedback by selecting seven different lateral-modes. The feedback strengths for different mode-selections are b), 21.6\%; c), $7.0 \%$; d), $4.8 \%$; e), $6.7 \%$; f), $9.9 \%$; and g), $11.6 \%$.

Figure 3 shows the intensity noise spectra of the BAL subjected to the lateral-mode-selected external feedback on perfect alignment condition, the noise spectra, from a) to g), correspond to the corresponding curves denoted with the same letter in Fig. 2. The intensity noise is neglected without external-cavity feedback, shown in curve a). When the external feedback is applied, the intensity noise is increased strongly for most of the cases except curve e), where the noise level is almost the same as freely running condition. When the 0 -order lateral-mode is selected, the peaks in the intensity noise spectrum with spacing around $135 \mathrm{MHz}$ are observed in curve b), corresponding to a beating frequency of double roundtrip external-cavity modes, i.e., $c / 4 L$, where $c$ is the speed of light, and $L$ is the optical length of the external feedback cavity ${ }^{10,21}$. When high-order lateral modes are selected, the peaks of the intensity noise spectra with spacing around $270 \mathrm{MHz}$ are originated from the beating between different single roundtrip external-cavity modes, the mode spacing of single roundtrip external-cavity modes is $c / 2 L^{10,21}$.

Recently, we found that when high-order lateral modes are selected, the external cavity is formed between the mirror stripe and a phase conjugate mirror (PCM) induced in the BAL by four-wave mixing ${ }^{21}$. Single roundtrip external-cavity modes can be excited in this external cavity, and the beating of these external-cavity modes are the reason for the observed noise spectra with peak spacing of is $c / 2 L$. For curve e), we believe the external cavity is also established between the feedback mirror and the BAL, but it seems only one external-cavity mode is excited, thus no peaks are observed in the noise spectrum. The peak spacing of double roundtrip external-cavity mode in noise spectrum has been observed in both narrow-stripe diode laser with ordinary feedback ${ }^{8}$ and phase-conjugate feedback ${ }^{22}$, and BAL with tilted feedback $^{12}$. In Ref. 8, a period-doubling bifurcation is responsible for the observation of peaks with spacing of $c / 4 L$. The similar bifurcation behaviors were also observed in other nonlinear feedback system ${ }^{23}$. In both Ref. 12 and 22, a PCM (in Ref. 12, a PCM is generated by four-wave mixing in the BAL) is responsible for the generation of double roundtrip external-cavity modes. In our case, when 0 -order lateral-mode is selected, the feedback is an on-axis feedback, (i.e., not a tilted feedback), thus a PCM cannot be induced in the BAL ${ }^{21}$. The reason of the excitation of double roundtrip external-cavity modes for 0 -order lateral mode selection is still under investigation. 


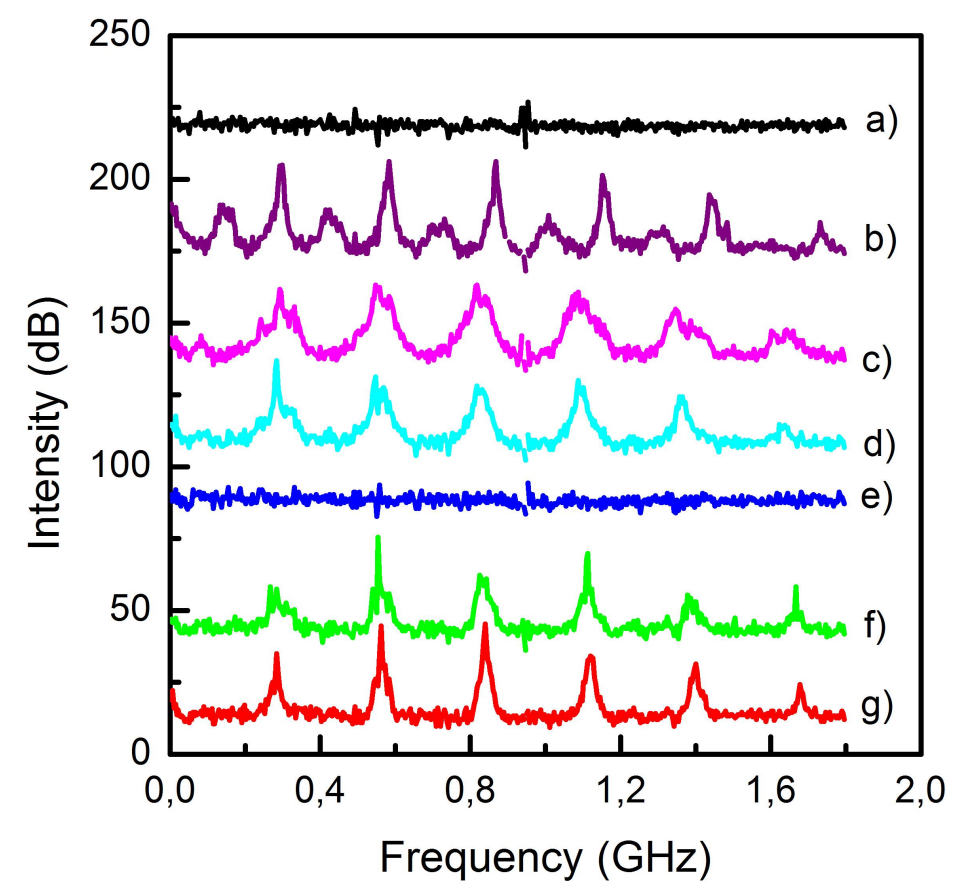

Figure 3. Intensity noise spectra of the output beam from the BAL without (black curve) and with (color curves) the lateralmode-selected external-cavity feedback. The curves in this figure correspond to the curves in Fig. 2 denoted with the same letter.

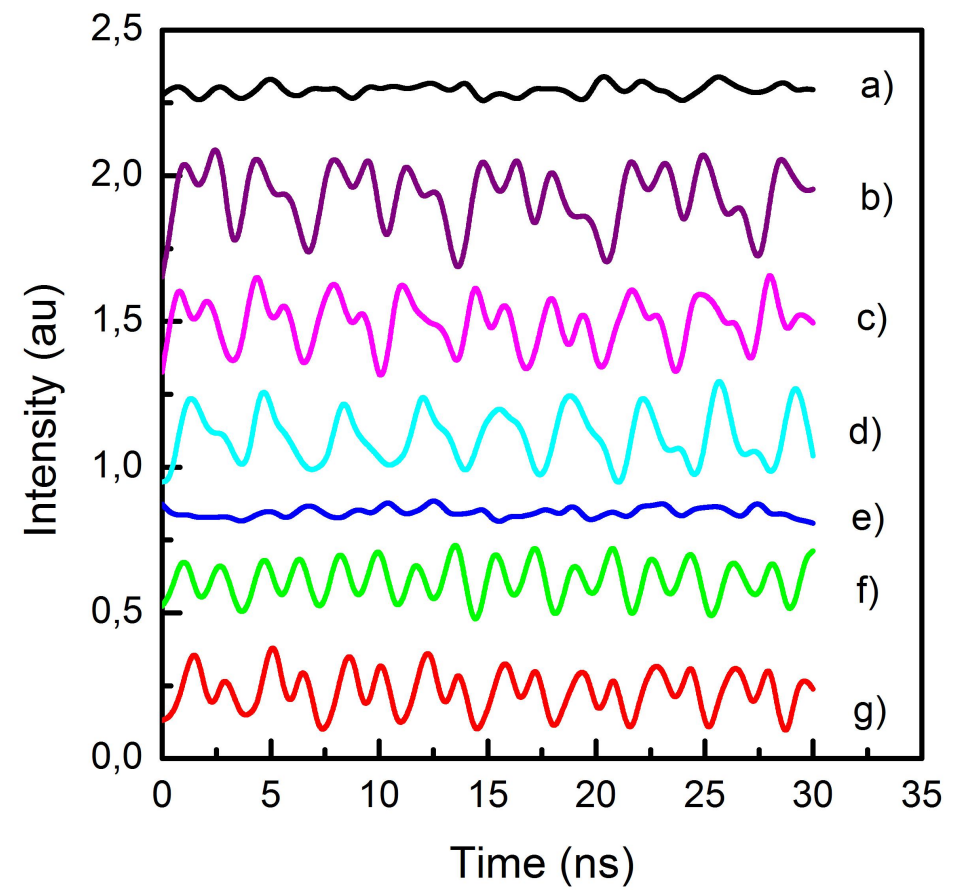

Figure 4. Time series of the output beam from the BAL without (black curve) and with (color curves) the lateral-modeselected external-cavity feedback. The curves in this figure correspond to the curves in Fig. 2 denoted with the same letter.

The corresponding time series of the output power with perfect lateral-mode-selected feedback are shown in Fig. 4. Compared with other curves, the fluctuation of curve a) and e) is quite small, and this is consistent with the noise spectra shown in Fig. 3. When other high-order lateral-modes are selected, periodic oscillations with the beat frequency of the adjacent single roundtrip external-cavity modes are observed $(c / 2 L)$, and high-frequency component with frequency of 
$c / L$ can also be observed in curves f) and $\mathrm{g}$ ). When the 0 -order lateral-mode is selected, the oscillated frequency of the output power corresponds to the beat frequency of the double roundtrip external-cavity modes, i.e., $c / 4 L$, is observed, and high-frequency components with the frequency of $c / 2 L$ and $c / L$ can also be observed. The time series shown in Fig. 4 are consistent with the intensity noise spectra shown in Fig. 3.

Recently, Pimenov et al studied the dynamical regimes of a multi-stripe laser array with external off-axis feedback theoretically ${ }^{20}$. Different periodic and irregular time dependences of the output intensity, such as power stable regime $(\mathrm{CW})$, periodic regime with one peak or two peaks (P1 or P2), aperiodic regime (A) and multimode periodic regime (PM), were shown in the study. Although a non-striped BAL is used in this study, we can still compare our results with the theory qualitatively since self-induced "stripes" can be formed in the transverse section of non-striped BAL with high-order lateral-mode selected external-feedback ${ }^{5}$. Other than the case of curve e) shown in Fig. 3 and Fig.4, the laser system is operated in both multiple longitudinal modes and multiple external-cavity modes when high-order lateralmode is selected, the periodic oscillation of the output power with a period of single external-cavity round trip time is similar to the case of multimode periodic regime (PM) in Ref. 20, but there are two peaks in each period for our case, this is similar to the case $\mathrm{P} 2$ in the theoretical study. Stable output with single external-cavity mode operation, like curve e), is also shown in the theory as $\mathrm{CW}$ regime ${ }^{20}$. The theoretical study does not include the condition of 0 -order lateral mode feedback (on-axis feedback), i.e., curve b) in our case.

When the mirror stripe is not aligned perfectly, the far-field profiles corresponding to two different lateral-mode selections are shown in Fig. 5, and for comparison, the far-field profiles on perfect alignment condition are also shown in the figure. Compared with the perfect alignment condition, the effects of the misalignment are a shorter output lobe and a little bit wide separation of the feedback and the output lobes, although the position of the mirror stripe is unchanged for both conditions. The feedback strengths on the non-perfect alignment condition are a little bit larger than that on the perfect alignment condition, this is consistent with the shorter output lobe on the non-perfect alignment condition as shown in the figure, since the ratio of the portion of the feedback lobe to the total beam profile is increased on the nonperfect alignment condition.

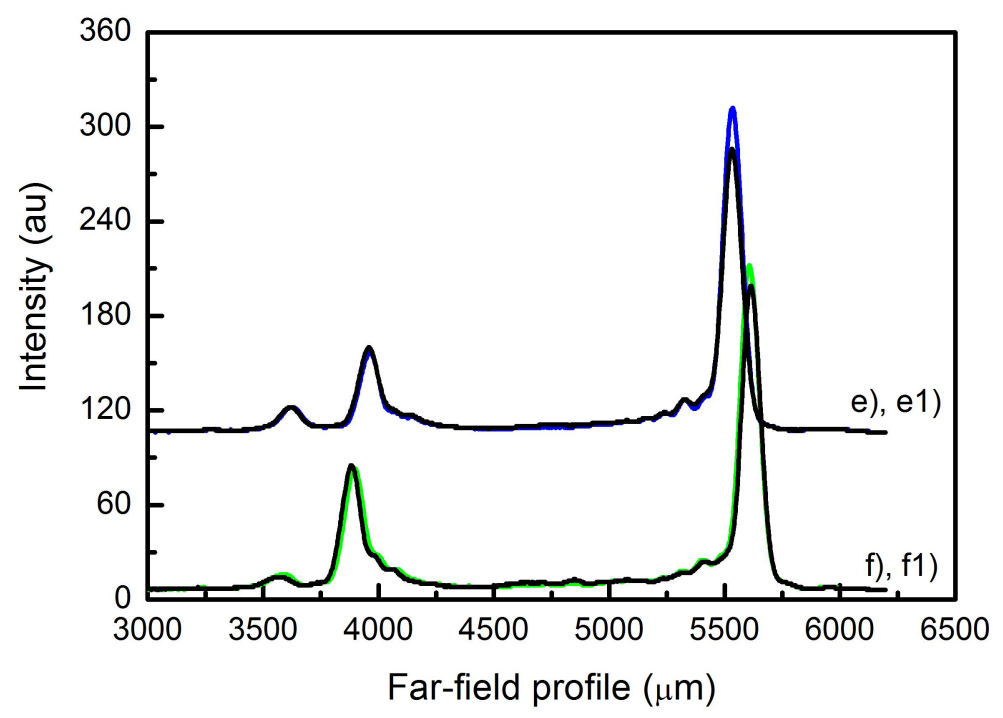

Figure 5. Far-field profiles of the BAL measured along the slow axis with perfect (color curves) and corresponding nonperfect (black curves) external-cavity feedback. The curves for perfect feedback here are the same as curves in Fig. 2 denoted with the same letter. The feedback strengths for the non-perfect condition are e1), $6.8 \%$; and f1), $10.1 \%$.

Figure 6 shows the corresponding intensity noise spectra for both the perfect and non-perfect alignment of the mirror stripe for the two lateral-mode selections. For the noise spectra for non-perfect alignment, other than the peaks of the beating frequencies of the single roundtrip external-cavity modes, $v_{\mathrm{EC}}$, a peak at round $108 \mathrm{MHz}$ for low frequency fluctuations, $v_{\mathrm{LFF}}$, is also observed. Additionally, in curves e1) and f1), we can also observe some peaks at the frequencies $v_{\mathrm{EC}} \pm v_{\mathrm{LFF}}$ generated by the mixing of external-cavity frequencies $v_{\mathrm{EC}}$ and LFF frequency $v_{\mathrm{LFF}}$. Here we should notice that the intensity noise spectra are similar for el) and f1), this is contrary to the perfect alignment condition. This means, in contrary to single external-cavity mode operation for e), the laser system is operated in 
multiple external-cavity modes for e1). Fig. 7 shows the time series of the output power for both perfect and non-perfect alignment conditions. The time series e1) and f1) show a periodic LFF oscillation consisted of the frequency components corresponding to the beating of external cavity modes.

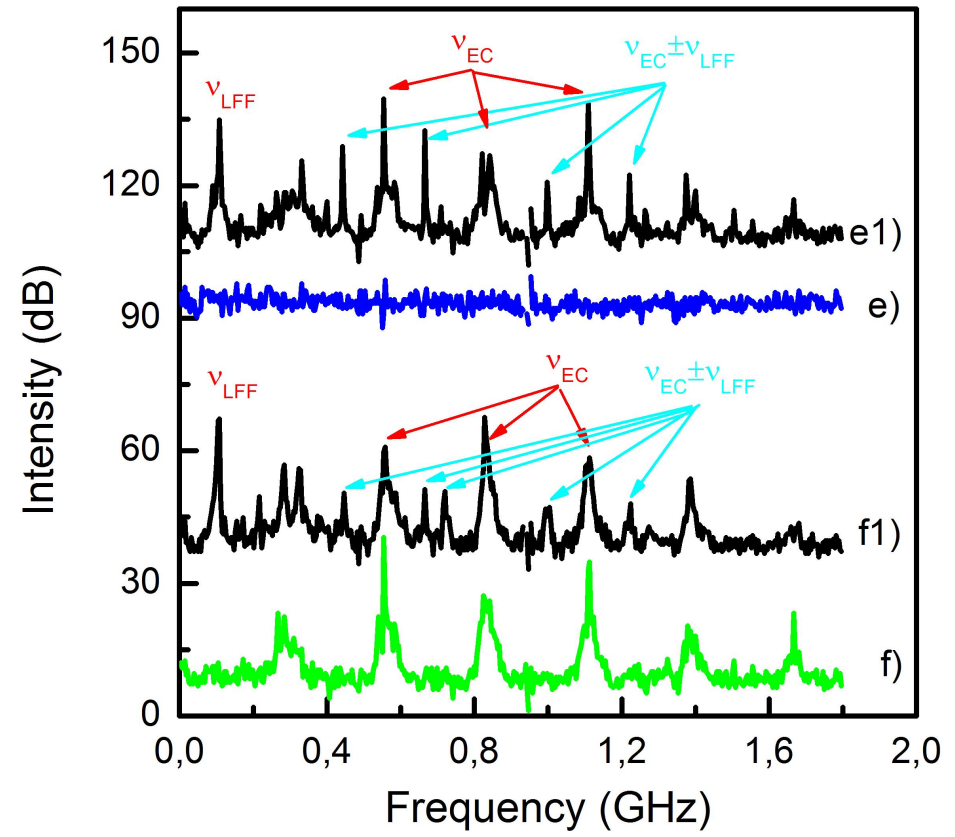

Figure 6. Intensity noise spectra of the output beam from the BAL with perfect (color curves) and corresponding non-perfect (black curves) external-cavity feedback. The curves for perfect feedback here are the same as the curves in Fig. 3 denoted with the same letter.

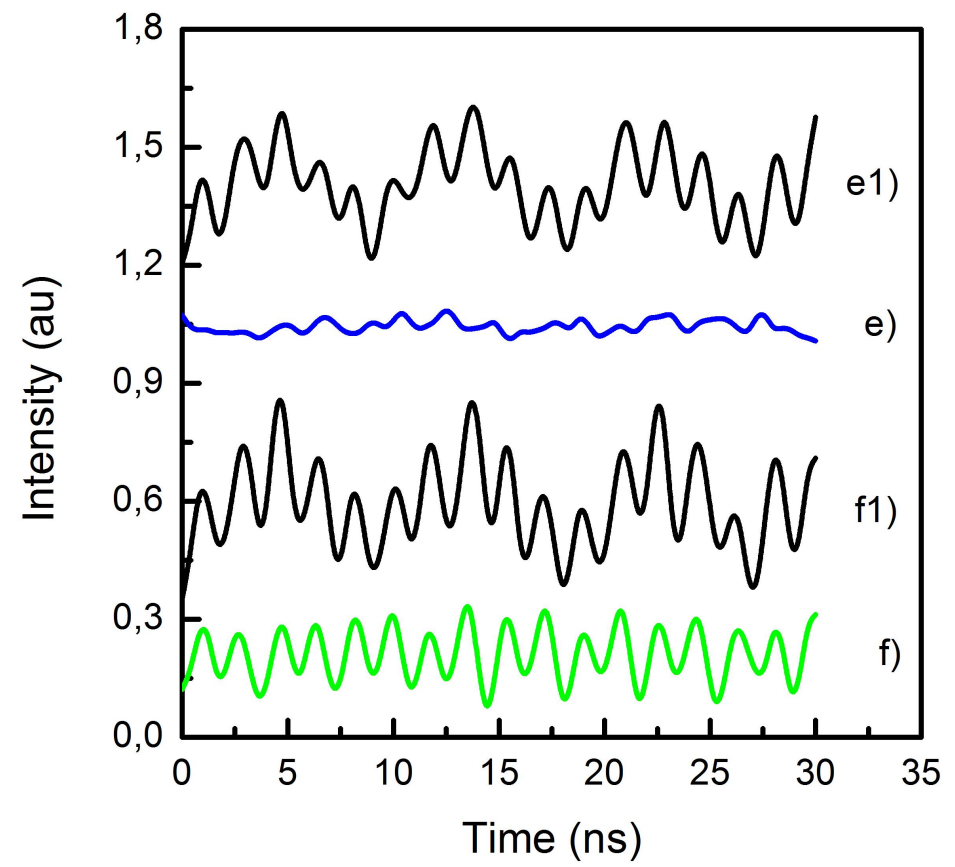

Figure 7. Time series of the output beam from the BAL with perfect (color curves) and corresponding non-perfect (black curves) external-cavity feedback. The curves for perfect feedback here are the same as the curves in Fig. 4 denoted with the same letter. 
Irregular LFFs have been observed frequently in diode lasers (both narrow stripe diode lasers and BALs) subjected to both conventional and phase-conjugate external feedback ${ }^{9,10,14,17}$. Regular LFFs have also been observed in diode laser (both narrow stripe diode lasers and BALs) with external optical feedback, but mainly in the short-cavity regime (i.e., the beating frequency of the external cavity modes is larger than the laser relaxation oscillation frequency) ${ }^{11,16,19}$, and rarely with long-cavity feedback (the beating frequency of the external cavity modes is less than the relaxation oscillation frequency) $)^{9}$. In the short-cavity regime, the regular LFF is named as pulse-package oscillation ${ }^{11,16,19}$, where periodic oscillation of the output power with the frequency of external-cavity loop is modulated by periodic LFFs; in the long-cavity regime, the regular LFF is named as synchronous Sisyphus effect ${ }^{9}$, where irregular fast pulses are modulated by periodic LFFs. The noise spectra and time series for the non-perfect alignment observed here show the regular LFFs consist of frequency components of external-cavity loop. This is the same as the pulse-package oscillation obtained for both narrow-stripe edge-emitting diode laser ${ }^{11}$ and BALs ${ }^{16,19}$. To our knowledge, this is the first time that pulse-package oscillation is observed in diode laser with the external feedback in long-cavity regime.

In principle, all the spatiotemporal dynamic behaviors shown in diode lasers are induced by the nonlinear interaction of the light field and the carrier in the gain medium of the diode laser, and the delayed light field generated by the external feedback may make the interaction more complex. On the non-perfect alignment condition, i.e., the feedback beam is coupled into the laser cavity with a little larger angle compared with the perfect alignment; a higher order lateral-mode may be excited to some extent. This is consistent with the fact that a little wider separation of the feedback lobe and the output lobe is observed compared with perfect alignment, as shown in Fig. 5. More complex dynamic behavior will be induced by the involvement of the higher order lateral-mode in the interaction between light field and the carrier, and by the competition between different lateral modes ${ }^{16}$. This may be the origin of the pulse-package oscillation observed on the non-perfect alignment condition.

\section{CONCLUSION}

In conclusion, the temporal dynamic behaviors of a BAL with lateral-mode-selected long-cavity feedback are investigated experimentally. When the feedback mirror stripe is perfectly aligned, by selecting different lateral modes, different dynamics, such as stable output, oscillated outputs with frequencies of both single roundtrip external-cavity loop and double roundtrip external-cavity loop, are observed. When the feedback mirror stripe is not aligned perfectly, pulse-package oscillation is observed, for the first time to our knowledge, in diode laser with external feedback in longcavity regime.

\section{REFERENCES}

[1] Wolff, S., Rodionov, A., Sherstobitov, V. E. and Fouckhardt, H., "Fourier-optical transverse mode selection in external-cavity broad-area lasers: experimental and numerical results," IEEE J. Quantum Electron. 39, 448-458 (2003).

[2] Chi, M., Thestrup, B. and Petersen, P. M., "Self-injection locking of an extraordinarily wide broad-area diode laser with a 1000- $\mu \mathrm{m}$-wide emitter," Opt. Lett. 30, 1147-1149 (2005).

[3] Lim, J. J., Sujecki, S., Lang, L., Zhang, Z., Paboeuf, D., Pauliat, G., Lucas-Leclin, G., Georges, P., Mackenzie, R. C. I., Bream, P., Bull, S., Hasler, K.-H., Sumpf, B., Wenzel, H., Erbert, G., Thestrup, B., Petersen, P. M., Michel, N., Krakowski, M. and Larkins, E. C., "Design and simulation of next-generation high-power, high-brightness laser diodes,” IEEE J. Sel. Top. Quantum Electron. 15, 993-1008 (2009).

[4] Jechow, A., Lichtner, M., Menzel, R., Radziunas, M., Skoczowsky, D. and Vladimirov, A. G., "Stripe-array diodelaser in an off-axis external cavity: theory and experiment," Opt. Express. 17, 19599-19604 (2009).

[5] Lichtner, M., Tronciu, V. Z. and Vladimirov, A. G., "Theoretical investigation of striped and non-striped broad area lasers with off-axis feedback," IEEE J. Quantum Electron. 48, 353-360 (2012).

[6] Verdiell, J.-M. and Frey, R., "A broad-area mode-coupling model for multiple-stripe semiconductor lasers," IEEE J. Quantum Electron. 26, 270-279 (1990).

[7] Pillai, R. M. R. and Garmire, E. M., "Paraxial-misalignment insensitive external-cavity semiconductor-laser array emitting near-diffraction limited single-lobed beam,” IEEE J. Quantum Electron. 32, 996-1008 (1996).

[8] Ye, J., Li, H. and McInerney, J. G., "Period-doubling route to chaos in a semiconductor laser with weak optical feedback," Phys. Review A 47, 2249-2252 (1993).

[9] Gavrielides, A., Newell, T. C., Kovanis, V., Harrison, R. G., Swanston, N., Yu, D. and Lu, W., "Synchronous Sisyphus effect in diode lasers subject to optical feedback," Phys. Review A 60, 1577-1581 (1999). 
[10] Lawrence, J. S. and Kane, D. M., "Contrasting conventional optical and phase-conjugate feedback in laser diodes," Phys. Review A 63, 033805 (2001).

[11]Heil, T., Fischer, I., Elsäßer, W. and Gavrielides, A., "Dynamics of semiconductor lasers subject to delayed optical feedback: the short cavity regime," Phys. Review Lett. 87, 243901 (2001).

[12] Vasil'ev P. P. and White, I. H., "Phase-conjugation broad area twin-contact semiconductor laser," Appl. Phys. Lett. 71, 40-42 (1997).

[13]Løbel, M., Petersen, P. M. and Johansen, P. M., "Suppressing self-induced frequency scanning of a phase conjugate diode laser array using counterbalance dispersion," Appl. Phys. Lett. 72, 1263-1265 (1998).

[14] Lawrence, J. S. and Kane, D. M., "Broad-area diode lasers with plane-mirror and phase-conjugate feedback," J. Lightwave Technol. 20, 100-104 (2002).

[15] Mandre, S. K., Fischer, I. and Elsässer, W., "Control of the spatiotemporal emission of a broad-area semiconductor laser by spatially filtered feedback," Opt. Lett. 28, 1135-1137 (2003).

[16] Mandre, S. K., Fischer, I. and Elsäßer, W., "Spatiotemporal emission dynamics of a broad-area semiconductor laser in an external cavity: stabilization and feedback-induced instabilities," Opt. Commun. 244, 355-365 (2005).

[17] Fujita Y. and Ohtsubo, J., "Optical-feedback-induced stability and instability in broad-area semiconductor lasers," Appl. Phys. Lett. 87, 031112 (2005).

[18] Tachikawa, T., Shogenji, R. and Ohtsubo, J., "Observation of multi-path interference in broad-area semiconductor lasers with optical feedback," Opt. Review 16, 533-539 (2009).

[19] Takeda, A., Shogenji, R. and Ohtsubo, J., "Dynamics and pulse-package oscillation in broad-area semiconductor lasers with short optical feedback," Appl. Phys. Lett. 101, 231105 (2012).

[20] Pimenov, A., Tronciu, V. Z., Bandelow, U. and Vladimirov, A. G., "Dynamical regimes of a multistripe laser array with external off-axis feedback,” J. Opt. Soc. Am. B 30, 1606-1613 (2013).

[21] Chi, M. and Petersen, P. M., "Spectral properties of a broad-area diode laser with off-axis external-cavity feedback," Appl. Phys. Lett. 103, 171112 (2013).

[22] Kürz, P. and Mukai, T., "Frequency stabilization of a semiconductor laser by external phase-conjugate feedback," Opt. Lett. 21, 1369-1371 (1996).

[23] Petersen, P. M., Ravn, J. N. and Skettrup, T., "Bifurcations in eletrooptic bistable devices with short delay times in the feedback," IEEE J. Quantum Electron. 20, 690-693 (1984). 